\title{
ChemComm
}

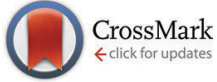

Cite this: Chem. Commun., 2016, 52,13714

DOI: $10.1039 / c 6 c c 90512 a$

\section{Correction: Dielectric constant enhancement of non-fullerene acceptors via side-chain modification}

\author{
Jenny E. Donaghey, Ardalan Armin, Dani M. Stoltzfus, Paul L. Burn* and \\ Paul Meredith*
}

Correction for 'Dielectric constant enhancement of non-fullerene acceptors via side-chain modification' by Jenny E. Donaghey et al., Chem. Commun., 2015, 51, 14115-14118.

www.rsc.org/chemcomm

Since publication of the original manuscript, improved procedures and purification methods have been developed for the preparation of M1, M2, and M3. These improvements are described in an additional ESI document which has been added to the original manuscript. The improved procedures and purifications have also led to the following corrections in the manuscript:

(1) It was found that $n$-octyl- $p$-toluenesulfonate was better to use than 1-bromooctane in the substitution reaction to form 7 . The text should therefore read "The side-chains were introduced to 2-bromofluorene and cyclopentadithiopehene (CPDT) via a nucleophilic substitution reaction with 1-bromo-2-(2-methoxyethoxy)ethane for M1 and M2, and $n$-octyl-p-toluenesulfonate for M3."

(2) A boronic acid rather than boronate ester was used in the Suzuki coupling. The text should therefore read "Functionalization of the 2-position of fluorene and CPDT with a boronic acid and tri- $n$-butylstannane, respectively, then enabled palladium catalysed couplings with 7-bromobenzothiadiazole-4-carbaldehyde."

(3) M1 and M2 were purified using different solvent systems. The text should therefore read "M1 was purified by precipitation from diethyl ether/light petroleum to afford a fluorescent orange powder. M2 was isolated as a blue powder by precipitation from a dichloromethane/light petroleum mixture and M3 was isolated as a viscous blue oil."

(4) The thermal transitions of $\mathbf{M} 2$ were found to be higher than first reported and $\mathbf{M} 3$ was found to have a $T_{\mathrm{g}}$. The text should therefore read "The same was observed for $\mathbf{M} 2$ which showed two melts at $129{ }^{\circ} \mathrm{C}$ and $143{ }^{\circ} \mathrm{C}$ on the first heating cycle suggesting the presence of two different polymorphs. At high scan rates $\left(100{ }^{\circ} \mathrm{C} \mathrm{min}^{-1}\right)$ a glass transition $\left(T_{\mathrm{g}}\right)$ could be observed for both molecules on the 2nd heating cycle at $11{ }^{\circ} \mathrm{C}$ for $\mathbf{M 1}$ and $19{ }^{\circ} \mathrm{C}$ for M2 (ESI). This means that once melted, the materials remain in an amorphous glassy state at room temperature. In comparison to K12, functionalization with glycol chains (M1) clearly has the effect of lowering the $T_{\mathrm{g}}$ and hindering crystallisation that can normally be induced via thermal annealing. ${ }^{16} \mathbf{M} 3$, which has longer alkyl chains, had a $T_{\mathrm{g}}$ at $13{ }^{\circ} \mathrm{C}$ in each heating scan."

(5) The solution UV-visble spectra were recorded in dichloromethane and the spectra of K12 and M1 are better described as being "essentially similar in solution and the solid-state". Similarly, the film spectra of $\mathbf{M} 2$ and $\mathbf{M} 3$ are similar in solution and the solid-state. The text should therefore read "The solution state absorption maxima of $\mathbf{M} 2$ and $\mathbf{M} 3$ are very similar. The optical gaps for $\mathbf{M} 2$ and $\mathbf{M} 3$ (Table 1) are identical suggesting that the introduction of glycol chains has little effect on the optical properties of the material. The solid-state absorption spectra of $\mathbf{M} 2$ and $\mathbf{M} 3$ are also similar." The solution and solid-state spectra can be seen in the corrected Fig. 2 and summarised in amended Table 1.

Table 1 Optical, thermal and electrochemical properties, static dielectric constants and electron mobilities of K12, M1, M2 and M3

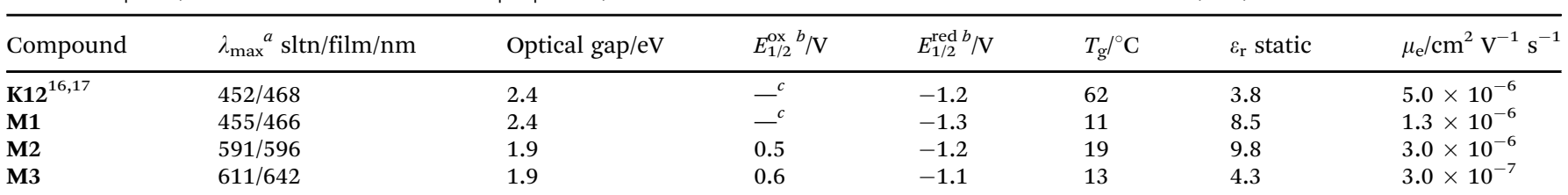

${ }^{a}$ In dichloromethane/spin-coated on fused silica from chloroform. ${ }^{b} \mathrm{~V} v s . \mathrm{Fc} / \mathrm{Fc}^{+} .{ }^{c}$ Oxidation outside solvent window. 
a)

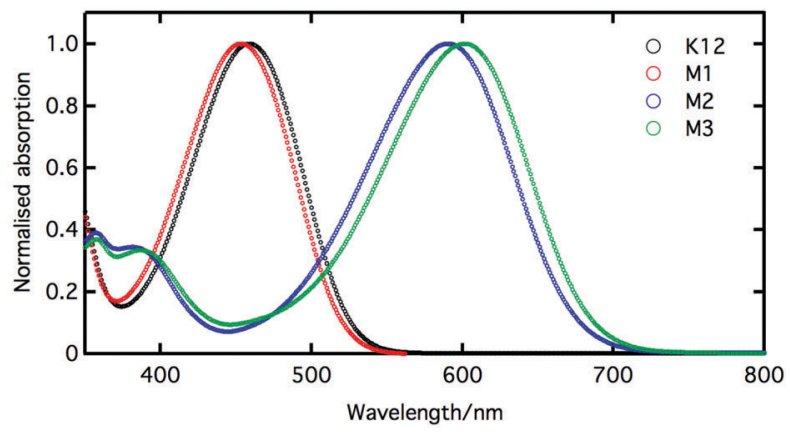

b)

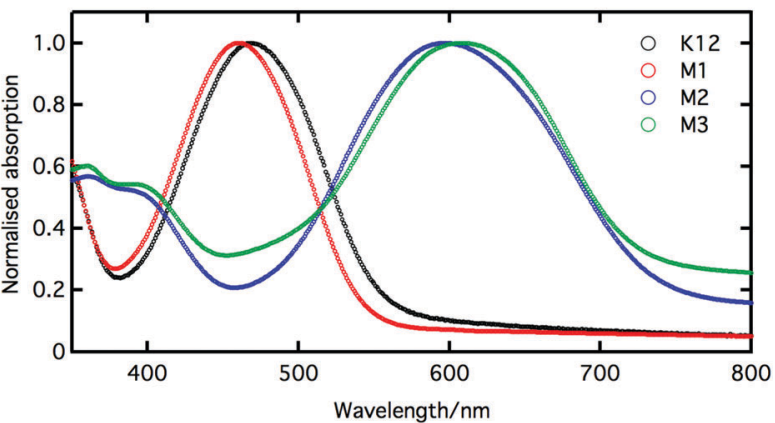

Fig. 2 Normalised UV-Vis absorption spectra of K12, M1, M2 and M3 in (a) dichloromethane; (b) thin film spin-coated from chloroform.

The Royal Society of Chemistry apologises for these errors and any consequent inconvenience to authors and readers. 